\title{
Transport of proteins into mitochondria
}

\author{
N. Pfanner and W. Neupert \\ Institute for Physiological Chemistry, The University of Munich, Munich, \\ Federal Republic of Germany
}

Current Opinion in Cell Biology 1989, 1:624-629

\section{Introduction}

More than $90 \%$ of mitochondrial proteins are encoded by nuclear genes and are synthesized as precursor proteins on cytosolic polysomes [1]. The translocation of mitochondrial proteins from the cytosol to their functional destination in one of the four mitochondrial subcompartments (outer membrane, intermembrane space, inner membrane and matrix; Fig. 1) involves a complex series of steps. The field of mitochondrial protein import has undergone very rapid development in recent years. Most studies have used fungal mitochondria (the yeast Saccharomyces cerevisiae and Neuraspora crassa) in vitro and in vivo. This development includes the characterization of properties of precursor proteins, the resolution of distinct translocation intermediates on the import pathways and the identification of components of the protein import machinery.

\section{Specific recognition of mitochondrial precursor proteins}

Mitochondrial precursor proteins contain positively charged targeting sequences. In many cases, targeting signals are found in $\mathrm{N}$-terminal peptide extensions of about

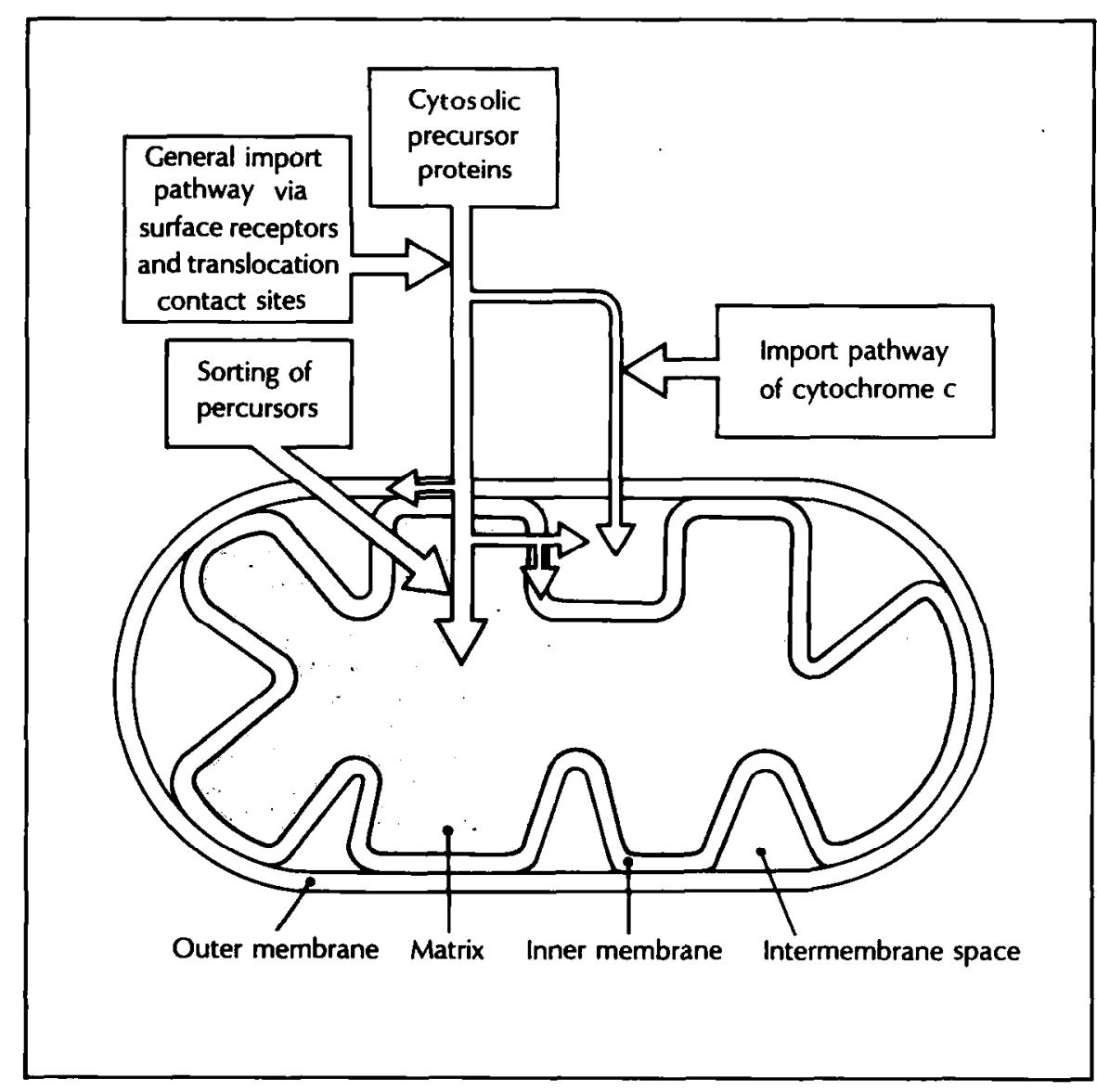

Fig. 1. Transport pathways of precursor proteins into mitochondria.

Abbreviations

GIP-general insertion protein; hsp60-heat-shock protein of 60 kD; PEP-processing enhancing protein. 
20-70 amino acid residues, termed presequences (Hurt and van Loon, Trends Biochem Sci 1986, 11:204-207) [2], which are cleaved off by specific peptidases inside the mitochondria. The main feature that mitochondrial targeting sequences have in common is the presence of positively charged amino acid residues and the nearly complete absence of negatively charged residues. In addition, targeting sequences may form amphiphilic structures under appropriate conditions [3]. So far, no particular amino acid sequence motif has been identified in the targeting peptides. This led to the suggestion that their secondary structure may have an important role in directing proteins into mitochondria. In several cases, the mature (non-cleavable) regions of precursor proteins may contribute to the specific high-affinity interaction of precursors with binding sites on the mitochondria, possibly via hydrophobic 'assistant sequences' [4].

Proteinaceous receptors on the mitochondrial surface are responsible for the specific recognition and high-affinity binding of precursor proteins [5,6] (Pfanner and Neupert, $J$ Biol Chem 1987, 262:7528-7536). In the current working model, at least two distinct receptor proteins are required for the binding of the various precursor proteins (Fig. 2) [6]. High-affinity binding sites could be reconstituted into liposomes, providing an important tool for the purification of receptor sites [5]. Antibodies which are directed against $45 \mathrm{kD}$ mitochondrial proteins inhibit import of precursor proteins, indicating that a $45 \mathrm{kD}$ protein could be involved in translocation of precursors (Ohba and Schatz, EMBO J 1987, 6:2109-2115).

A detailed analysis of the role of the surface receptors gave rise to an explanation for a previously surprising result. Non-mitochondrial signals, such as chloroplast signal sequences, are able to direct proteins into mitochondria, albeit with a low efficiency (Hurt et ah, EMBO J1986, 5:1343-1350). It was observed that precursor proteins can bypass the proteinaceous surface receptors with a very low efficiency, i.e. can enter the mitochondrial import pathway at a later stage (Pfaller et al, $J$ Biol Cbem 1989, 264:34-39). This bypass transport appears to be the only pathway used by non-mitochondrial signals. The very low import rates make it likely that bypass import does not disturb the selectivity of mitochondrial protein uptake and the unique mitochondrial protein composition [7].

\section{Translocation of precursors into and across the mitochondrial membranes}

Precursor proteins with a stable tertiary structure cannot be inserted into mitochondrial membranes (Eilers and Schatz, Nature 1986, 322:228-232). Precursors are at least partially unfolded before, or during, membrane translocation. This can be clearly demonstrated by the reversible accumulation of precursors spanning both mitochondrial membranes. The $\mathrm{N}$-terminal portion of the precursor enters the matrix space, whereas a (folded) $\mathrm{C}$. terminal region remains in the cytosol (Schleyer and Neupert, Cell 1985, 43:339-350) [8]. Moreover, destabiliza- tion of the tertiary structure of precursor proteins ('unfolding'), e.g. by the introduction of point mutations, can increase the rates of import into mitochondria [9].

Import of mitochondrial precursor proteins requires hydrolysis of ATP (Pfanner and Neupert, FEBS Lett 1986, 209:152-156; for a summary, see [10]). ATP is involved in the generation or maintenance of a translocation-competent (loosely folded) precursor conformation. In particular, incompletely folded polypeptide chains require less ATP for import than their more strongly folded counterparts [11]. Translocation into the outer mitochondrial membrane of a chemically denaturated (unfolded) precursor protein is completely independent of ATP [12]. Since the specific import criteria, such as high-affinity binding to surface receptors, are fulfilled by the chemically unfolded precursor [5], the role of ATP seems to be (directly or indirectly) related to modulation of the conformation of precursors. Yeast mutants which are defective in a subset of stress proteins ('heat-shock proteins') of the $70 \mathrm{kD}$ class have been shown to be deficient in import of some mitochondrial proteins [13]. Seventykilodalton stress proteins are generally thought to be involved in modification of the conformation of proteins in an ATP-dependent manner [14]. In fact, Murakami et al [15] demonstrated that $70 \mathrm{kD}$ stress proteins, and a further cytosolic component, stimulate transport of a precursor protein into mitochondria in vitro. The present working model includes the participation of $70 \mathrm{kD}$ stress proteins, and possibly other factors, in the generation or maintenance of a transport-competent conformation of precursor proteins. It seems unlikely that cytosolic factors also have a role in other steps of mitochondrial protein import, such as targeting of precursors, since several precursors can be imported in the absence of any cytosolic factor $[5,10]$.

The outer mitochondrial membrane contains apparently a common membrane insertion site used by all precursor proteins studied so far with the exception of cytochrome $c$ (Fig. 2). This site, which is saturable with precursor proteins, is termed the 'general insertion protein' (GIP) [6]. Insertion into the outer membrane seems to require a high degree of unfolding of precursors and relatively high levels of ATP (Pfanner et al, Cell 1987, 49:815-823). After interaction with GIP, the precursors are inserted into the inner membrane. This step depends on the electrical potential across the inner membrane; the potential (negative inside) may exert an electrophoretic effect on the positively charged targeting sequences (Pfanner and Neupert, EMBO J 1985, 4:2819-2825) [3]. Butow and colleagues (Kellems et al, J Cell Biol 1975, 65:1-14) proposed that proteins are translocated at morphologically visible sites of close contact between mitochondrial outer and inner membranes. The experimental proof for translocation of precursor proteins via contact sites was provided by the demonstration of reversible trapping of precursors in contact sites (Schleyer and Neupert, 1985). Contact sites are stable structures which can be enriched in submitochondrial vesicles (Schwaiger et al, J Cell Biol 1987, 105:235-246). Precursor proteins which are inserted into contact sites are extracted from the membranes by pro- 


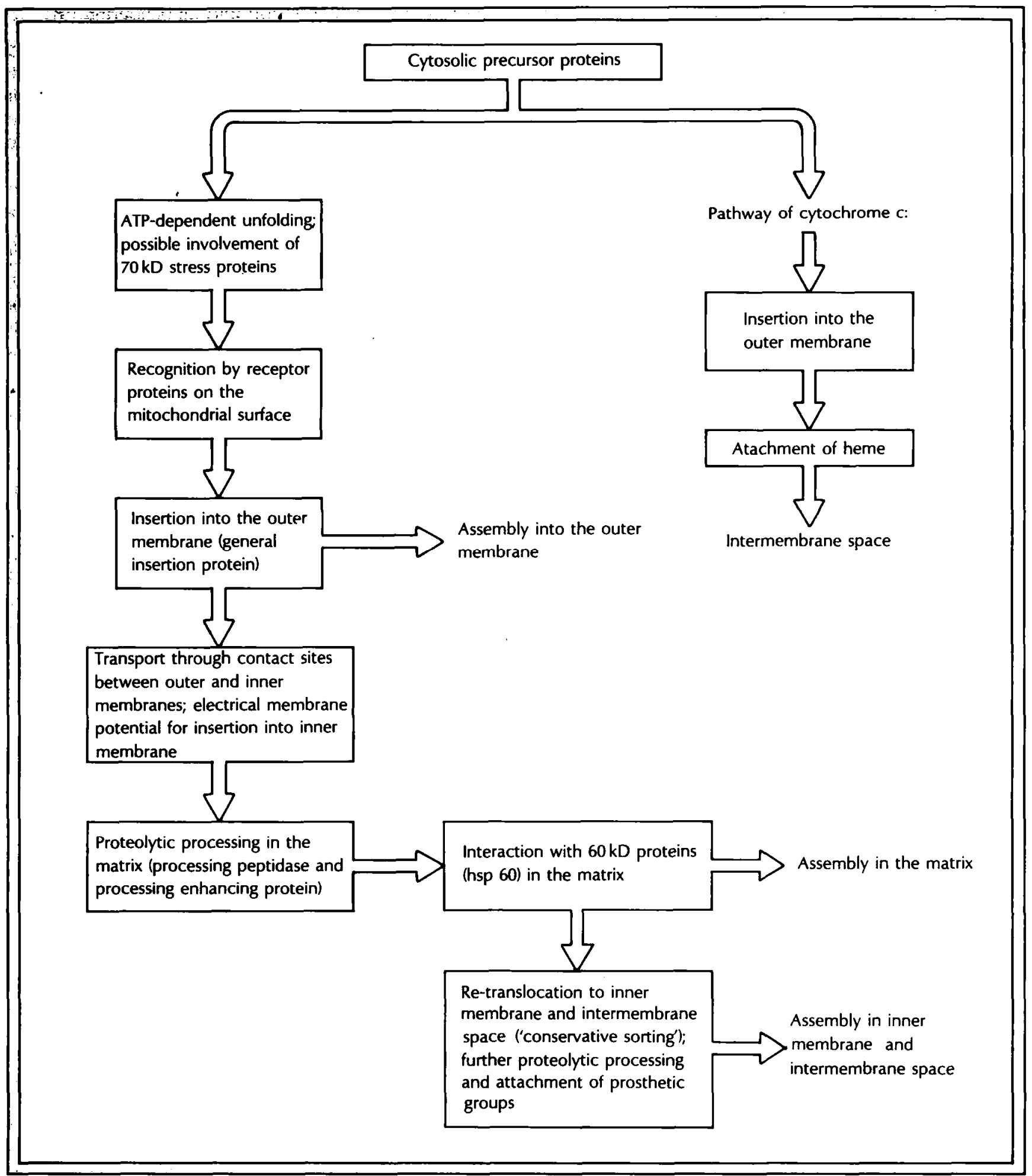

Fig. 2. Mechanisms and steps of mitochondrial protein import.

tein denaturants such as urea or at alkaline $\mathrm{pH}[16]$. The contact sites can be saturated by membrane-spanning precursors [17]. In summary, proteinaceous components seem to be of great importance for both the structure and the function of contact sites.

\section{Processing and folding of imported precursors}

The positively charged targeting sequence (presequence) is proteolytically cleaved off by the processing peptidase in the mitochondrial matrix. The processing pep- 
tidase was purified and was found to be a soluble protein of about $57 \mathrm{kD}$ [18]. A protein of about $52 \mathrm{kD}$ which is mainly associated with the inner mitochondrial membrane greatly enhances the processing activity; it is termed the 'processing enhancing protein' (PEP) [18]. However, the processing peptidase and PEP do not form a detectable complex. PEP may bind precursor proteins protruding into the matrix space and then present them to the processing peptidase. Two previously described yeast mutants which are defective in maturation of mitochondrial precursor proteins were found to be deficient in processing peptidase and PEP, respectively (Yaffe and Schatz, Proc Natl Acad Sci USA 1984, 81:4819-4823) $[19,20]$. The biochemical purification and characterization of these two components of the mitochondrial import machinery thus allowed the determination of the functional defect in the two mutants. Processing peptidase and PEP have significant homology in their primary sequences, suggesting that they may have originated from a common ancestor $[20,21]$. Several precursor proteins undergo a second proteolytic cleavage step either in the mitochondrial matrix or in the intermembrane space [1]. The proteases which perform these processing steps have not yet been identified.

Recently, a new procedure for the selection of yeast mutants affected in mitochondrial protein import was worked out by testing the functional assembly of a nuclear-encoded matrix protein [20]. Several new mutants were found in addition to the two mutants described above. One of the mutants is defective in a constitutively expressed stress protein ('heat-shock protein') of about $60 \mathrm{kD}$ (hsp60) [22]. Hsp60 is homologous to the chloroplast ribulose 1,5-bisphosphate carboxylase (Rubisco) subunit-binding protein and the Escberichia coli heat-shock protein groEL [23] (Hemmingsen et al., $\mathrm{Na}$ ture 1988, 333:330-334). These three proteins are named 'chaperonins', since they apparently ensure proper protein interaction during the assembly of oligomeric protein complexes. Hsp60 interacts with precursor proteins that are transported into the mitochondrial matrix; this step is a prerequisite for assembly of the precursors into protein complexes and for the further transport of precursors into, or across, the inner mitochondrial membrane (see below). Hsp60 may act by conferring a conformation on precursors which is required for assembly or intramito. chondrial sorting [22]. The action of a stress protein in the mitochondrial matrix may represent a second ATP. dependent step in mitochondrial protein import, in addition to the ATP-dependent unfolding of cytosolic precursors.

\section{Intramitochondrial sorting of precursors}

Precursor proteins destined for the outer mitochondrial membrane or the mitochondrial matrix follow relatively simple sorting pathways (Fig. 1,2). Outer membrane proteins interact with a specific receptor on the mitochondrial surface, insert into the membrane, with the help of GIP, and then become assembled in the outer membrane. Matrix proteins also interact with specific recep- tors and with GIP and are then translocated through contact sites into the matrix [1].

Precursor proteins which are destined for the intermem brane space or the inner membrane follow more elaborate sorting pathways (Figs. 1, 2). Most of them also use surface receptors, GIP and contact sites for transport into the matrix. After proteolytic processing and interaction with hsp 60 , the precursors are redirected into or across the inner membrane (Hartl et al., Cell 1986, 47:939-951) [24]. The mechanisms of retranslocation from the matrix space into or across the inner mitochondrial membrane resemble the transport mechanisms in prokaryotes, the ancestors of mitochondria. This led to the following model of intramitochondrial sorting ('conservative sorting') [24]. The mitochondrial genes that originally encoded mitochondrial proteins were transferred to the nucleus and acquired a segment coding for a positively charged presequence. The presequence directs the precursors through contact sites into the mitochondrial matrix. After the first proteolytic cleavage, which results in the removal of the positively charged targeting sequence, the precursor is released into its ancestral folding and assembly pathway. Several precursors carry a second targeting signal in the C-terminal half of the presequence. This relatively hydrophobic signal strongly resembles prokaryotic leader sequences which direct the export of prokaryotic proteins [1]. In fact, the second targeting signal seems to direct translocation of precursors across the inner mitochondrial membrane, and is cleaved off at the intermembrane space side of the membrane. Originally, it was proposed that the second (hydrophobic) signal sequence acts as a 'stop-transfer sequence' by preventing the further translocation of a precursor protein into the matrix (van Loon and Schatz, EMBO J 1987, 6:2441-2448); the precursor protein would then reach its location in the inner membrane or the intermembrane space by lateral diffusion in the inner membrane. As discussed above, this model does not apply to the sorting pathways of precursor proteins studied so far. However, the possibility that some precursors of integral inner membrane proteins may follow such a pathway cannot be excluded.

Cytochrome $c$, a protein of the intermembrane space, uses an import pathway which is quite distinct from the pathways of the other mitochondrial precursor proteins. The precursor protein, apocytochrome $c$, can insert into the outer mitochondrial membrane without the aid of surface receptors or GIP $[6,25]$. Cytochrome $c$ heme lyase, a protein of the intermembrane space which is responsible for the covalent addition of heme to the apoprotein, seems to have a crucial role in the translocation of cytochrome $c$ into the intermembrane space. Mutants which are defective in cytochrome $c$ heme lyase are deficient in binding and import of cytochrome $c$ [26] (Dumont et al., EMBO J 1987, 6:235-241). Apocytochrome $c$ may spontaneously insert into the outer membrane and binds with high affinity, forming a complex which includes heme lyase. After addition of heme, the holoprotein is released into the intermembrane space [25]. C-terminal portions of the precursor protein are ap- 
parently important for targeting of apocytochrome $c$ to mitochondria (Stuart et al, EMBOJ 1987, 6:2131-2137).

Why are several (often very hydrophobic) proteins synthesized within mitochondria, although most mitochondrial proteins are encoded by nuclear genes? One possible explanation is that such hydrophobic proteins can. not be translocated through the cytosol and the two mitochondrial membranes, or that they would not be able to assume the correct orientation and conformation for assembly when imported from outside the organelle. Nagley et al. [27] excluded these possibilities using an elegant approach. They replaced the mitochondrial gene for a membrane protein by an artificial nuclear gene that also contained a segment encoding a presequence. The protein was successfully imported into mitochondria and functionally assembled into a multi-subunit complex. Hence, the most likely reason for the lack of transfer of the remaining mitochondrial genes to the nucleus appears to be the divergence of the genetic code.

\section{Conclusions}

Rapid progress in the field of mitochondrial protein import led to the identification of several components of the import machinery by biochemical and genetic approaches. The generation and characterization of defined translocation intermediates on the import pathways of precursor proteins opens the way for the identification of more components. The conformation of precursor proteins is important for membrane translocation and assembly of proteins. Elucidation of the functional and structural properties of mitochondrial precursor proteins and of the transport components is not only essential for the understanding of mitochondrial biogenesis, but is also important for the unravelling of intracellular protein transport and biogenesis of cell organelles in general.

\section{Acknowledgements}

We thank R.A Stuan for critical reading of the manuscript.

\section{Annotated references and recommended reading}

- Of interest

$\bullet \quad$ Of outstanding interest

1. Hartl. f.U, Pfanner N, Nicholson DW, neupert W: Mi-

- tochondrial protein import. Biocbim Biopbys Acta 1989, 988:1-45.

Very recent, comprehensive review of mitochondrial protein import.

2. HoRVICH AL, Kalousek F, Fenton WA, Furtak K, POLLOCK

- RA, ROSENBERG IE: The ornithine transcarbamylase leader peptide directs mitochondrial import through both its midportion structure and net positive charge. J Cell Biol 1987, 105:669-678.

A net positive charge is essential for the function of this presequence, as is the case for most mitochondrial targeting sequences. With this precursor protein, the midportion of the presequence is sufficient for targeting, whereas in many other presequences the N-terminal half carries sufficient targeting information.

3. ROISE D, SCHATZ G: Mitochondrial presequences. J Biol - Chem 1988, 263:4509-4511.

A minireview of structure and function of mitochondrial targeting sequences.

4. Pfanner N, MUlefr HK, Harmey MA, Neupert W: Mitochon-

- drial protein import: involvement of the mature part of a cleavable precursor protein in the binding to receptor sites. $F M B O J 1987,6: 3449-3454$

Hydrophobic 'assistant-sequences' in the mature part of some precursor proteins can - in addition to the effect of hydrophilic presequences - increase the affinity for binding sites on the mitochondrial surface, and thereby increase the rate of import.

5. PFAllfR $R$, NeUPERT W: High-affinity binding sites involved - in the import of porin into mitochondria. EMBO $J 1987$, 6:2635-2642

Preparation of large amounts of a pure precursor protein allows quantitation of high-affinity binding sites on the mitochondrial surface. The binding sites are reconstituted into liposomes. Import of this (unfolded) precursor is independent of cytosolic cofactors.

6. Pfaller R, Steger hF, Rassow J, Pfanner N, Neupert W: - Import pathways of precursor proteins into mitochondria: multiple receptor sites are followed by a common membrane insertion site. J Cell Biol 1988, 107:2483-2490.

Competition of various precursor proteins for mitochondrial import sites reveals the existence of a common membrane insertion site, GIP, in the outer membrane which is used by precursors destined for different mitochondrial subcompartments. Distinct receptor sites precede GIP in the pathway.

7. Pfanner N, Pfauer R, Neupert W: How finicky is mitochon- drial protein import? Trends Biochem Sci 1988, 13:165-167. Discusses mitochondrial protein import as a multi-step system. Some of the specific steps, such as binding to receptor sites, can be bypassed with a very low efficiency. This provides an explanation for the surprising result that non-mitochondrial signal sequences can direct proteins into mitochondria.

8. CHEN WJ, DOUGLAS MG: The role of protein structure in - the mitochondrial import pathway. Unfolding of mitochondrially bound precursors is required for membrane trans. location. J Biol Chem 1987, 262:15605-15609.

A hybrid precursor protein with a folded C.terminal part is trapped in contact sites between both mitochondrial membranes, suggesting that unfolding on the mitochondrial surface is required for completion of translocation.

9. VeSTXYeBER D, SCHATZ G: Point mutations destabilizing a - precursor protein enhance its post-translational import into mitochondria. EMBO J 1988, 7:1147-1152.

The tertiary structure of a precursor protein is destabilized by sitedirected mutagenesis, leading to an increased rate and efficiency of im. port

10. Ellers M, SCHATZ G: Ptotein unfolding and the energetics - of protein translocation across biological membranes. Cell $1988,52: 481-483$.

Minireview of the energetics of protein translocation and its relation to unfolding of precursor proteins. Provides a very interesting comparison of the mechanisms used for translocation of proteins across various biological membranes.

11. VERNER K, SCHATZ G: Import of an incompletely folded - precursor protein into isolated mitochondria requires an energized inner membrane, hut no added ATP. EMBO $J$ 1987, 6:2449-2456.

Incompletely synthesized (nascent) chains of precursors are imported into mitochondria. These loosely folded polypeptides require less ATP for import than the corresponding complete (and folded) precursor proteins.

12. Pfanner $N$, Pfaller $R$, Kieene $R$, Ito $M$, tropschug $M$,

- NEUPERT W: Role of ATP in mitochondrial protein import. 
Conformational alteration of a precursor protein can substitute for ATP requirement. J Biol Chem 1988, 263:4049-4051. An arificially unfolded precursor of a mitochondrial outer membrane protein is specifically imported into mitochondria. Its import is com. pletely independent of ATP, suggesting that the ATP-dependence of protein import is related to modifications of the precursor conforma. tion.

13. Deshaies RJ, KOCH BD, Werner-WashbuRNe M, Craig WJ, - SCHEKMAN R: A subfamily of stress proteins facilitates translocation of secretory and mitochondrial precursor polypeptides. Nature 1988, 332:800-804.

Yeast mutants which are depleted of a subset of $70 \mathrm{kD}$ stress proteins are deficient in import of precursor proteins into the endoplasmic retic ulum and mitochondria. $70 \mathrm{kD}$ stress proteins thus may participate in both transport pathways.

14. Pelmam H: Heat-shock proteins: coming in from the cold - $\quad$ Nature 1988, 332:776-77.

Brief discussion of the role of heat shock proteins in intracellular pro tein traffic.

15. Murakami H, Pain D, Blobel G: $70 \cdot \mathrm{kD}$ heat shock-related - protein is one of at least two distinct cytosolic factors stimulating protein import into mitochondria. J Cell Biol 1988, 107:2051-2058.

Identification of a $70 \mathrm{kD}$ stress protein as a cytosolic factor stimulating transport of a precursor protein into mitochondria in vitro. In addition, evidence for a second cytosolic factor which acts synergistically with the stress protein is presented.

16. Pfanner N, Hartl F-U, Guiard B, Neupert W: Mitochondrial - precursor proteins are imported through a hydrophilic membrane environment. Eur J Biochem 1987, 169:289-293. Mitochondrial precursor proteins which accumulate in contact sites in a 2-membrane spanning fashion are extractable with urea or at alkaline $\mathrm{pH}(\mathrm{pH} 11.5)$. Extraction by protein denaturants suggests that the precursors were in a hydrophilic (proteinaceous) membrane environment.

17. VESTWEBER D, SCHATZ G: A chimeric mitochondrial precur- sor protein with internal disulfide bridges blocks import of authentic precursors into mitochondria and allows quantitation of import sites. I Cell Biol 1988, 107:2037-2044

A precursor protein with a stably folded C.terminal portion is irreversibly trapped in mitochondrial contact sites. In this way, import of other precursor proteins is blocked and the number of translocation contact sites is assessed.

18. HAWUTSCHEK G. SCHNEIDER H, SCHMIDT B, TROPSCHUG $M$. •• HARTL F.U, NEUPERT W: Mitochondrial protein import: identification of processing peptidase and of PEP, a processing enhancing protein. Cell 1988, 53:795-806.

Identification and functional characterization of the first 2 components of the mitochondrial protein import machinery. The processing pepti. dase which cleaves imported precursors is a soluble protein of the mi. tochondrial matrix, whereas PEP, which greatly stimulates proteolytic processing, is largely associated with the inner membrane.

19. YANG M, JENSEN RE, YAFFE MP, OPPLGER W, SCHATZ G: Im- port of proteins into yeast mitochondria: the purified matrix processing protease contains two subunits which are encoded by the nuclear MASI and MAS2 genes. EMBO J $1988,7: 3857-3862$.

2 previously described yeast mutants which are defective in maturation of mitochondrial precursor proteins are found to be deficient in processing peptidase and PEP, respectively.

20. Pollock RA, Harth F-U, Cheng MT, OSTERmanN J, HORWICH

- A, NEUPERT W: The processing peptidase of yeast mitochondria: the two co-operating components MPP and PEP are structurally related. $E M B O J 1988,7: 3493-3500$.
Describes a new strategy for isolation of yeast mutants affecting import and/or assembly of mitochondrial proteins. 1 of the mutants is deficient in processing peptidase. The primary sequence of processing peptidase is homologous to that of PEP.

21. JENSEN RE, YAFFE MP: Import of proteins into yeast mito-

- chondria: the nuclear MAS2 gene encodes a component of the processing protease that is homologous to the MAS1encoded subunit. EMBO J 1988, 7:3863-3872.

Processing peptidase and PEP have homologous primary sequences.

22. Cheng MY, Hartl F.U, Martin J, POLOCK RA, KaLOUSEK F, - NEUPERT W, HAJUBERG EM, HAUIBERG RL, NORWICH AI. Mitochondrial heat-shock protein hsp60 is essential for assembly of proteins imported into yeast mitochondria. Nature 1989, 337:620-624.

Hsp60, which is located in the mitochondrial matrix, is required for assembly of precursor proteins. A yeast mutant deficient in hsp 60 is also affected in the retranslocation of precursor proteins from the matrix into or across the inner membrane [24].

23. ReADING DS, HallBerg RL, MYers AM: (Letter to the Editor) - Characterization of the yeast HSP6O gene coding for 2 mitochondrial assembly factor. Nature 1989, 337:655-658.

The primary sequence of yeast mitochondria hsp 60 is homologous to that of the $E$. coli groEL protein and the Rubisco subunit-binding protein of chloroplasts. The 3 proteins ('chaperonins') facilitate assembly of protein complexes.

24. Hartl F-U, Ostermann J, Guiard B, Neupert W: Succes-. Sive translocation into and out of the mitochondrial matrix: targeting of proteins to the intermembrane space by a bipartite signal peptide. Cell 1987, 51:1027-1037.

Several precursor proteins which are destined for the mitochondrial intermembrane space are first completely imported into the matrix and are then redirected across the inner membrane. The principles of translocation from the matrix across the inner membrane seem to be similar to the mechanisms of protein export in prokaryotic cells, the ancestors of mitochondria. The sorting pathway via the matrix space is thus called 'conservative sorting'.

25. NiChOLSON DW, Hergersberg C, NEUPERT W: Role of cy- tochrome $c$ heme lyase in the import of cytochrome $c$ into mitochondria. I Biol Chem 1988, 263:19034-19042.

A translocation intermediate is accumulated where the precursor of cy. tochrome $c$ spans the outer mitochondrial membrane and forms a tight complex with the cytochrome $c$ heme lyase. After covalent attachment of heme, cytochrome $c$ is completely translocated into the intermembrane space.

26. Nargang FE, Drygas ME, KwONG PL, Nicholson DW, NeUPERT - W: A mutant of Neumspora crassa deficient in cytochrome $c$ heme lyase activity cannot import cytochrome $c$ into mitochondria. $J$ Biol Chem 1988, 263:9388-9394.

Mitochondria from a $N$. crassa mutant which is defective in cytochrome $c$ heme lyase activity are deficient in binding and import of apocytochrome $c$, suggesting that cytochrome $c$ heme lyase has an important role in the import pathway of cytochrome $c$.

27. Naglfy P, Farrell LB, Gearing DP, Nero D, Meltzer S, - DEVENISH RJ: Assembly of functional proton-translocating ATPase complex in yeast mitochondria with cytoplasmically synthesized subunit 8 , a polypeptide normally encoded within the organelle. Proc Natl Acad Sci USA 1988, 85:2091-2095.

A hydrophobic mitochondrial membrane protein which is normally synthesized within mitochondria is expressed from an artificial nuclear gene which also codes for a presequence. The protein is imported into mitochondria and correctly assembled into a muldi-subunit complex. This elegant approach demonstrates that very hydrophobic proteins can also be imported from the cytosol. 